Salim A. Messaoudi • Abdelfeteh Fareh

\title{
Energy decay in a Timoshenko-type system of thermoelasticity of type III with different wave-propagation speeds
}

Received: 26 July 2012 / Accepted: 4 December 2012 / Published online: 9 January 2013

(C) The Author(s) 2012. This article is published with open access at Springerlink.com

Abstract In this paper, we consider a one-dimensional linear Timoshenko system of thermoelasticity type III and prove a polynomial stability result for the non-equal wave-propagation speed case.

Mathematics Subject Classification 35B37 · 35L55 - 74D05 · 93D15 · 93D20

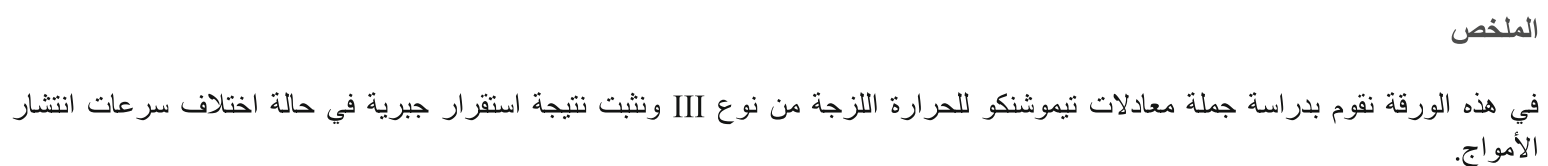

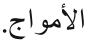

\section{Introduction}

A simple model describing the transverse vibration of a beam, which has the form

$$
\left\{\begin{array}{l}
\rho u_{t t}=\left(K\left(u_{x}-\varphi\right)\right)_{x}, \quad \text { in }(0, L) \times(0,+\infty) \\
I_{\rho} \varphi_{t t}=\left(E I \varphi_{x}\right)_{x}+K\left(u_{x}-\varphi\right), \text { in }(0, L) \times(0,+\infty),
\end{array}\right.
$$

was developed by Timoshenko [24], where $t$ denotes the time variable and $x$ is the space variable along the beam of length $L$, in its equilibrium configuration, $u$ is the transverse displacement of the beam and $\varphi$ is the rotation angle of the filament of the beam. The coefficients $\rho, I_{\rho}, E, I$ and $K$ are respectively the density (the mass per unit length), the polar moment of inertia of a cross section, Young's modulus of elasticity, the moment of inertia of a cross section, and the shear modulus.

Many researchers got interested in studying (1.1) and various damping mechanisms have been used to stabilize the vibrations of this system. The obtained results show that the presence of dissipation for both equations leads to uniform stability (exponential or polynomial) regardless to the values of the constants $\rho, I_{\rho}, E, I$ and $K$. This has been demonstrated by Kim and Renardy [10], Feng et al. [3], Raposo et al. [21], Santos [22], Messaoudi and Mustafa [11] and others.

S. A. Messaoudi $(\varangle)$

Department of Mathematics and Statistics, KFUPM, Dhahran 31261, Saudi Arabia

E-mail: messaoud@kfupm.edu.sa

A. Fareh

Departement de Mathématiques, Faculté des Sciences et de Technologie,

Université d' El Oued,

B.P. 789, El Oued 39000, Algérie

E-mail: farehabdelf@gmail.com 
However, the stability in the case of only one damping in the second equation of (1.1) depends on the values of the constants $\rho, I_{\rho}, E, I$ and $K$. Precisely, if the wave-propagation speeds are equal $\left(\frac{K}{\rho}=\frac{E I}{I_{\rho}}\right)$, then a uniform stability can be obtained for weak solutions. This has been showed by Soufyane and Wehbe [23], Ammar-Khodja et al. [1], Guesmia and Messaoudi [8,9], Messaoudi and Mustafa [12,14] and Messaoudi et al. [13], Fernández Sare and Rivera [4], Messaoudi and Said-Houari [16], Rivera and Racke [18,19] and Mustafa and Messaoudi [20]. Whereas, in the opposite case $\left(\frac{K}{\rho} \neq \frac{E I}{I_{\rho}}\right)$, a weaker rate of decay is obtained for more regular solutions. In this regard, we quote, among others, the work of Fernández Sare and Rivera [4], Messaoudi and Said-Houari [16].

Regarding stabilization via heat effect, Rivera and Racke [17] considered the following system

$$
\left\{\begin{array}{l}
\rho_{1} \varphi_{t t}-\left(\sigma\left(\varphi_{x}, \psi\right)\right)_{x}=0 \\
\rho_{2} \psi_{t t}-b \psi_{x x}+k\left(\varphi_{x}+\psi\right)+\gamma \theta_{x}=0 \\
\rho_{3} \theta_{t}-k \theta_{x x}+\gamma \psi_{t x}=0
\end{array}\right.
$$

where $\varphi, \psi$, and $\theta$ are the transverse displacement of the beam, the rotation angle of the filament and the difference temperature, respectively. Under appropriate conditions of $\sigma, \rho_{i}, b, k, \gamma$, they proved several exponential decay results for the linearized system and non-exponential stability result for the case of different wave speeds.

In the above system, the heat flux is given by Fourier's law. As a result, this theory predicts an infinite speed of heat propagation. That is any thermal disturbance at one point has an instantaneous effect elsewhere in the body. Experiments showed that heat conduction in some dielectric crystals at low temperatures is free of this paradox and disturbances, which are almost entirely thermal, propagate in a finite speed. To overcome this physical paradox, many theories have merged such as thermoelasticity by second sound or thermoelasticity type III. For background related to this theory, we refer the reader to Green and Naghdi [5-7] and the review paper of Chandrasekharaiah [2].

Messaoudi and Said-Houari [15] considered the following Timoshenko-type system with thermoelasticity type III

$$
\left\{\begin{array}{l}
\rho_{1} \varphi_{t t}-K\left(\varphi_{x}+\psi\right)_{x}=0 \quad \text { in }(0, \infty) \times(0,1), \\
\rho_{2} \psi_{t t}-b \psi_{x x}+K\left(\varphi_{x}+\psi\right)+\beta \theta_{x}=0 \quad \text { in }(0, \infty) \times(0,1), \\
\rho_{3} \theta_{t t}-\delta \theta_{x x}+\beta \psi_{t t x}-\kappa \theta_{t x x}=0 \quad \text { in }(0, \infty) \times(0,1),
\end{array}\right.
$$

together with initial and boundary conditions, and showed, under the condition $\frac{K}{\rho_{1}}=\frac{b}{\rho_{2}}$, that weak solutions decay exponentially. In the present work, we consider (1.2) for the case $\frac{K}{\rho_{1}} \neq \frac{b}{\rho_{2}}$ and prove a polynomial decay result for strong solutions.

\section{Main result}

In this section, we state and prove our main decay result. In order to exhibit the dissipative nature of System (1.2), we introduce the new variables $\phi=\varphi_{t}$ and $\Psi=\psi_{t}$, So, System (1.2) takes the form

$$
\left\{\begin{array}{l}
\rho_{1} \phi_{t t}-K\left(\phi_{x}+\Psi\right)_{x}=0 \quad \text { in }(0, \infty) \times(0,1) \\
\rho_{2} \Psi_{t t}-b \Psi_{x x}+K\left(\phi_{x}+\Psi\right)+\beta \theta_{t x}=0 \quad \text { in }(0, \infty) \times(0,1) \\
\rho_{3} \theta_{t t}-\delta \theta_{x x}+\beta \Psi_{t x}-\kappa \theta_{t x x}=0 \text { in }(0, \infty) \times(0,1) .
\end{array}\right.
$$

We supplement (2.1) with the following initial and boundary conditions

$$
\left\{\begin{array}{l}
\phi(., 0)=\phi_{0}, \phi_{t}(., 0)=\phi_{1}, \Psi(., 0)=\Psi_{0}, \Psi_{t}(., 0)=\Psi_{1} \\
\theta(., 0)=\theta_{0}, \theta_{t}(., 0)=\theta_{1} \\
\phi_{x}(0, t)=\phi_{x}(1, t)=\Psi(0, t)=\Psi(1, t)=\theta_{x}(0, t)=\theta_{x}(1, t)=0 .
\end{array}\right.
$$

From Eqs. (2.1) $1,(2.1)_{3}$ and (2.2), we easily verify that

$$
\frac{\mathrm{d}^{2}}{\mathrm{~d} t^{2}} \int_{0}^{1} \phi(x, t) \mathrm{d} x=0 \quad \text { and } \quad \frac{d^{2}}{\mathrm{~d} t^{2}} \int_{0}^{1} \theta(x, t) \mathrm{d} x=0 .
$$


So, if we set

$$
\begin{aligned}
& \bar{\phi}(x, t)=\phi(x, t)-t \int_{0}^{1} \phi_{1}(x) \mathrm{d} x-\int_{0}^{1} \phi_{0}(x) \mathrm{d} x \\
& \bar{\theta}(x, t)=\theta(x, t)-t \int_{0}^{1} \theta_{1}(x) \mathrm{d} x-\int_{0}^{1} \theta_{0}(x) \mathrm{d} x
\end{aligned}
$$

then simple substitution shows that $(\bar{\phi}, \Psi, \bar{\theta})$ satisfies (2.1), the boundary conditions in (2.2), and more importantly

$$
\int_{0}^{1} \bar{\phi}(x, t) \mathrm{d} x=0 \quad \text { and } \quad \int_{0}^{1} \bar{\theta}(x, t) \mathrm{d} x=0, \quad \forall t \geq 0 .
$$

In this case, Poincaré's inequality is applicable for $\bar{\theta}$ and $\bar{\phi}$. In the sequel, we work with $\bar{\phi}$ and $\bar{\theta}$ but for convenience, we write $\phi$ and $\theta$ instead.

Before stating and proving our stability result, we present a short discussion of the well-posedness and the semigroup formulation of (2.1) and (2.2). For this purpose, we introduce, as in [17],

$$
\begin{aligned}
& L_{*}^{2}(0,1)=\left\{u \in L^{2}(0,1) / \int_{0}^{1} u(x) \mathrm{d} x=0\right\} \\
& H_{*}^{1}(0,1)=\left\{u \in H^{1}(0,1) / \int_{0}^{1} u(x) \mathrm{d} x=0\right\}
\end{aligned}
$$

So, $V:=\left(\phi, \phi_{t}, \Psi, \Psi_{t}, \theta, \theta_{t}\right)^{T}$ satisfies

$$
\left\{\begin{array}{l}
V_{t}=A V \\
V(0)=V_{0}
\end{array}\right.
$$

where $V_{0}:=\left(\phi_{0}, \phi_{1}, \Psi_{0}, \Psi_{1}, \theta_{0}, \theta_{1}\right)^{T}$ and $A$ is the differential operator

$$
A=\left(\begin{array}{cccccc}
0 & 1 & 0 & 0 & 0 & 0 \\
\frac{K}{\rho_{1}} \partial_{x}^{2} & 0 & \frac{K}{\rho_{1}} \partial_{x} & 0 & 0 & 0 \\
0 & 0 & 0 & 1 & 0 & 0 \\
-\frac{K}{\rho_{2}} \partial_{x} & 0 & \frac{b}{\rho_{2}} \partial_{x}^{2}-\frac{K}{\rho_{2}} & 0 & 0 & -\frac{\beta}{\rho_{2}} \partial_{x} \\
0 & 0 & 0 & 0 & 0 & 1 \\
0 & 0 & 0 & -\frac{\beta}{\rho_{3}} \partial_{x} & \frac{\delta}{\rho_{3}} \partial_{x x} & \frac{\kappa}{\rho_{3}} \partial_{x x}
\end{array}\right) .
$$

The energy space

$$
\mathcal{H}:=H_{*}^{1}(0,1) \times L_{*}^{2}(0,1) \times H_{0}^{1}(0,1) \times L^{2}(0,1) \times H_{*}^{1}(0,1) \times L_{*}^{2}(0,1)
$$

is a Hilbert space with respect to the inner product

$$
\begin{aligned}
\langle V, W\rangle_{\mathcal{H}}= & \rho_{1}\left\langle V^{2}, W^{2}\right\rangle_{L^{2}(0,1)}+\rho_{2}\left\langle V^{4}, W^{4}\right\rangle_{L^{2}(0,1)}+b\left\langle V_{x}^{3}, W_{x}^{3}\right\rangle_{L^{2}(0,1)} \\
& +K\left\langle V_{x}^{1}+V^{3}, W_{x}^{1}+W^{3}\right\rangle_{L^{2}(0,1)}+\delta\left\langle V^{5}, W^{5}\right\rangle_{L^{2}(0,1)}+\rho_{3}\left\langle V^{6}, W^{6}\right\rangle_{L^{2}(0,1)}
\end{aligned}
$$

for $V=\left(V^{1}, V^{2}, V^{3}, V^{4}, V^{5}, V^{6}\right)^{T}$ and $W=\left(W^{1}, W^{2}, W^{3}, W^{4}, W^{5}, W^{6}\right)^{T}$. The domain of $A$ is then

$$
\mathcal{D}(A):=\left\{V \in \mathcal{H} / V^{1}, V^{3}, V^{5} \in H^{2}(0,1) / V_{x}^{1}, V^{4}, V_{x}^{5}, V_{x}^{6} \in H_{0}^{1}(0,1), V^{2} \in H_{*}^{1}(0,1)\right\} .
$$


Theorem 2.1 Assume that $\left(\phi_{0}, \phi_{1}, \Psi_{0}, \Psi_{1}, \theta_{0}, \theta_{1}\right) \in D(A)$. Then, (2.1) and (2.2) have a unique strong solution satisfying

$$
\left(\phi, \phi_{t}, \Psi, \Psi_{t}, \theta, \theta_{t}\right) \in C^{1}([0, \infty), \mathcal{H}) \cap C^{0}([0, \infty), \mathcal{D}(A)) .
$$

The proof can be carried similarly to [13].

To state our decay result, we introduce the first and second-order energy functionals:

$$
\begin{aligned}
& E_{1}(t)=\int_{0}^{1}\left(\rho_{1} \phi_{t}^{2}+\rho_{2} \Psi_{t}^{2}+\rho_{3} \theta_{t}^{2}+K\left|\phi_{x}+\Psi\right|^{2}+b \Psi_{x}^{2}+\delta \theta_{x}^{2}\right) \mathrm{d} x \\
& E_{2}(t)=\int_{0}^{1}\left(\rho_{1} \phi_{t t}^{2}+\rho_{2} \Psi_{t t}^{2}+\rho_{3} \theta_{t t}^{2}+K\left|\phi_{x t}+\Psi_{t}\right|^{2}+b \Psi_{x t}^{2}+\delta \theta_{x t}^{2}\right) \mathrm{d} x
\end{aligned}
$$

Theorem 2.2 Assume that $\left(\phi_{0}, \phi_{1}, \Psi_{0}, \Psi_{1}, \theta_{0}, \theta_{1}\right) \in D(A)$, then the strong solution (2.4) satisfies, for a positive constant $k$, independent of $t$ and the initial data, the estimate

$$
E_{1}(t) \leq \frac{k\left(E_{1}(0)+E_{2}(0)\right)}{t}, \quad \forall t>0
$$

The proof of our result will be established through several lemmas.

Lemma 2.3 Let $(\phi, \Psi, \theta)$ be the strong solution of (2.1) and (2.2). Then, we have

$$
\begin{aligned}
& E_{1}^{\prime}(t)=-\kappa \int_{0}^{1} \theta_{t x}^{2} \mathrm{~d} x \leq 0 \\
& E_{2}^{\prime}(t)=-\kappa \int_{0}^{1} \theta_{t t x}^{2} \mathrm{~d} x \leq 0 .
\end{aligned}
$$

Proof Multiplying equations in (2.1) by $\phi_{t}, \Psi_{t}$ and $\theta_{t}$, respectively, integrating over $(0,1)$ and summing up we obtain (2.6). Then, differentiating (2.1) with respect to $t$ and multiplying the resulting equations by $\phi_{t t}, \Psi_{t t}$ and $\theta_{t t}$, respectively, integrating over $(0,1)$ and summing up we obtain $(2.7)$.

Lemma 2.4 Let $(\phi, \Psi, \theta)$ be the strong solution of (2.1) and (2.2). Then, the functional

$$
I_{1}(t):=\rho_{2} \int_{0}^{1} \Psi_{t} \Psi-\rho_{1} \int_{0}^{1} \phi_{t}\left(\int_{0}^{x} \Psi(y, t) \mathrm{d} y\right)
$$

satisfies, for all $\varepsilon_{1}>0$,

$$
I_{1}^{\prime}(t) \leq-\frac{b}{2} \int_{0}^{1} \Psi_{x}^{2}+\varepsilon_{1} \int_{0}^{1} \phi_{t}^{2}+\left(\rho_{2}+\frac{\rho_{1}^{2}}{4 \varepsilon_{1}}\right) \int_{0}^{1} \Psi_{t}^{2}+\frac{\beta^{2}}{2 b} \int_{0}^{1} \theta_{t x}^{2} .
$$

Proof By taking a derivative of $I_{1}$ and using (2.1) and (2.2), we conclude

$$
I_{1}^{\prime}(t)=-b \int_{0}^{1} \Psi_{x}^{2}+\rho_{2} \int_{0}^{1} \Psi_{t}^{2}-\beta \int_{0}^{1} \Psi \theta_{t x}-\rho_{1} \int_{0}^{1} \phi_{t}\left(\int_{0}^{x} \Psi_{t}(y, t) \mathrm{d} y\right) .
$$

Using Young's inequality and

$$
\left(\int_{0}^{x} \Psi_{t}(y, t) \mathrm{d} y\right)^{2} \leq \int_{0}^{1} \Psi_{t}^{2} \text { and } \int_{0}^{1} \Psi^{2} \leq \int_{0}^{1} \Psi_{x}^{2},
$$

Estimate (2.8) is established. 
Lemma 2.5 Let $(\phi, \Psi, \theta)$ be the strong solution of (2.1) and (2.2). Then, the functional

$$
I_{2}(t):=\rho_{2} \rho_{3} \int_{0}^{1} \Psi_{t}\left(\int_{0}^{x} \theta_{t}(y, t) \mathrm{d} y\right)-\delta \rho_{2} \int_{0}^{1} \theta_{x} \Psi
$$

satisfies, for all $\varepsilon_{2}>0$,

$$
I_{2}^{\prime}(t) \leq-\frac{\beta \rho_{2}}{2} \int_{0}^{1} \Psi_{t}^{2}+\varepsilon_{2} \int_{0}^{1}\left(\Psi_{x}^{2}+\phi_{x}^{2}\right)+C_{\varepsilon_{2}} \int_{0}^{1} \theta_{t x}^{2} .
$$

Proof By taking a derivative of $I_{2}$ and using (2.1) and (2.2), we get

$$
\begin{aligned}
I_{2}^{\prime}(t)= & \beta \rho_{3} \int_{0}^{1} \theta_{t}^{2}-\rho_{3} b \int_{0}^{1} \theta_{t} \Psi_{x}+\rho_{3} K \int_{0}^{1} \theta_{t} \phi-K \int_{0}^{1} \Psi\left(\int_{0}^{x} \theta_{t}(y, t) \mathrm{d} y\right) \\
& +\kappa \rho_{2} \int_{0}^{1} \theta_{t x} \Psi_{t}-\beta \rho_{2} \int_{0}^{1} \Psi_{t}^{2}-\delta \rho_{2} \int_{0}^{1} \Psi \theta_{t x} .
\end{aligned}
$$

The assertion of the lemma then follows, using Young's and Poincaré's inequalities.

Lemma 2.6 Let $(\phi, \Psi, \theta)$ be the strong solution of (2.1) and (2.2). Then, the functional

$$
I_{3}(t):=\rho_{2} \int_{0}^{1} \Psi_{t}\left(\phi_{x}+\Psi\right)+\frac{b \rho_{1}}{K} \int_{0}^{1} \Psi_{x} \phi_{t}
$$

satisfies

$$
I_{3}^{\prime}(t) \leq-\frac{K}{2} \int_{0}^{1}\left(\phi_{x}+\Psi\right)^{2}+\rho_{2} \int_{0}^{1} \Psi_{t}^{2}+\frac{\beta^{2}}{2 K} \int_{0}^{1} \theta_{t x}^{2}+\left(\frac{b \rho_{1}}{K}-\rho_{2}\right) \int_{0}^{1} \Psi_{t x} \phi_{t} .
$$

Proof A differentiation of $I_{3}$, taking into account (2.1) and (2.2), gives

$$
I_{3}^{\prime}(t)=-K \int_{0}^{1}\left(\phi_{x}+\Psi\right)^{2}-\beta \int_{0}^{1}\left(\phi_{x}+\Psi\right) \theta_{t x}+\rho_{2} \int_{0}^{1} \Psi_{t}^{2}+\left(\frac{b \rho_{1}}{K}-\rho_{2}\right) \int_{0}^{1} \Psi_{t x} \phi_{t} .
$$

Consequently, (2.10) follows by Young's inequality.

Lemma 2.7 Let $(\phi, \Psi, \theta)$ be the strong solution of (2.1) and (2.2). Then, the functional

$$
I_{4}(t):=-\rho_{1} \int_{0}^{1} \phi_{t} \phi-\rho_{2} \int_{0}^{1} \Psi_{t} \Psi
$$

satisfies

$$
\begin{aligned}
I_{4}^{\prime}(t) \leq & -\rho_{1} \int_{0}^{1} \phi_{t}^{2}-\rho_{2} \int_{0}^{1} \Psi_{t}^{2}+\left(b+\frac{1}{2}\right) \int_{0}^{1} \Psi_{x}^{2} \\
& +K \int_{0}^{1}\left(\phi_{x}+\Psi\right)^{2}+\frac{\beta^{2}}{2} \int_{0}^{1} \theta_{t x}^{2} .
\end{aligned}
$$


Proof A differentiation of $I_{4}$, taking into account (2.1) and (2.2), gives

$$
I_{4}^{\prime}(t)=-\rho_{1} \int_{0}^{1} \phi_{t}^{2}-\rho_{2} \int_{0}^{1} \Psi_{t}^{2}+b \int_{0}^{1} \Psi_{x}^{2}+K \int_{0}^{1}\left(\phi_{x}+\Psi\right)^{2}+\beta \int_{0}^{1} \Psi \theta_{t x} .
$$

Using Young's and Poincaré's inequalities for the last term, (2.11) follows.

Lemma 2.8 Let $(\phi, \Psi, \theta)$ be the strong solution of (2.1) and (2.2). Then, the functional

$$
I_{5}(t):=\int_{0}^{1} \rho_{3} \theta_{t} \theta+\frac{\kappa}{2} \theta_{x}^{2}+\beta \Psi_{x} \theta
$$

satisfies, for all $\varepsilon_{2}>0$,

$$
I_{5}^{\prime}(t) \leq-\delta \int_{0}^{1} \theta_{x}^{2}+\left(\rho_{3}+\frac{\beta^{2}}{4 \varepsilon_{2}}\right) \int_{0}^{1} \theta_{t}^{2}+\varepsilon_{2} \int_{0}^{1} \Psi_{x}^{2}
$$

Proof A simple differentiation of $I_{5}$, taking into account (2.1) and (2.2), leads to

$$
I_{5}^{\prime}(t)=\rho_{3} \int_{0}^{1} \theta_{t}^{2}-\delta \int_{0}^{1} \theta_{x}^{2}+\beta \int_{0}^{1} \Psi_{x} \theta_{t}
$$

Finally, by Young's inequality, (2.12) is obtained.

Proof of Theorem 2.2 We define the Lyapunov functional $\mathcal{L}$ as follows

$$
\mathcal{L}(t):=N\left(E_{1}(t)+E_{2}(t)\right)+N_{1} I_{1}+N_{2} I_{2}+I_{3}(t)+\frac{1}{4} I_{4}(t)+I_{5}(t) .
$$

A combination of (2.6)-(2.12) and use of

$$
\int_{0}^{1} \theta_{t}^{2} \leq \int_{0}^{1} \theta_{t x}^{2}, \quad \int_{0}^{1} \phi_{x}^{2} \mathrm{~d} x \leq 2 \int_{0}^{1}\left(\phi_{x}+\Psi\right)^{2}+2 \int_{0}^{1} \Psi_{x}^{2},
$$

give

$$
\begin{aligned}
\mathcal{L}^{\prime}(t) \leq & -\left(\frac{1}{4} \rho_{1}-N_{1} \varepsilon_{1}\right) \int_{0}^{1} \phi_{t}^{2}-\left(\frac{K}{4}-2 \varepsilon_{2} N_{2}\right) \int_{0}^{1}\left(\phi_{x}+\Psi\right)^{2} \\
& -\left[\frac{N_{2} \beta \rho_{2}}{2}-N_{1}\left(\rho_{2}+\frac{\rho_{1}^{2}}{4 \varepsilon_{1}}\right)-\frac{3}{4} \rho_{2}\right] \int_{0}^{1} \Psi_{t}^{2} \\
& -\left[\frac{N_{1} b}{2}-3 \varepsilon_{2} N_{2}-\frac{1}{4}\left(\frac{1}{2}+b\right)-\varepsilon_{2}\right] \int_{0}^{1} \Psi_{x}^{2}-\delta \int_{0}^{1} \theta_{x}^{2} \\
& -\int_{0}^{1} \theta_{t}^{2}-N \kappa \int_{0}^{1} \theta_{x t t}^{2}-(N \kappa-\lambda) \int_{0}^{1} \theta_{x t}^{2}+\left(\frac{b \rho_{1}}{K}-\rho_{2}\right) \int_{0}^{1} \Psi_{t x} \phi_{t}
\end{aligned}
$$

where $\lambda$ is a positive constant independent of $N$. 
At this point, we choose our constants carefully. First, let us take $N_{1}$ large enough such that $\frac{N_{1} b}{4}-$ $\frac{1}{4}\left(\frac{1}{2}+b\right)>0$, then pick $\varepsilon_{1}$ so small that $\frac{1}{4} \rho_{1}-N_{1} \varepsilon_{1}>0$. We then choose $N_{2}$ large enough so that $\frac{N_{2} \beta \rho_{2}}{2}-N_{1}\left(\rho_{2}+\frac{\rho_{1}^{2}}{4 \varepsilon_{1}}\right)-\frac{3}{4} \rho_{2}>0$. Finally, we select $\varepsilon_{2}$ so small that

$$
\frac{N_{1} b}{2}-3 \varepsilon_{2} N_{2}-\frac{1}{4}\left(\frac{1}{2}+b\right)-\varepsilon_{2}>0 \text { and } \quad \frac{K}{4}-2 \varepsilon_{2} N_{2}>0 .
$$

Therefore (2.14) takes the form

$$
\mathcal{L}^{\prime}(t) \leq-2 \eta E_{1}(t)-N \kappa \int_{0}^{1} \theta_{x t t}^{2}-(N \kappa-\lambda) \int_{0}^{1} \theta_{x t}^{2}+\left(\frac{b \rho_{1}}{K}-\rho_{2}\right) \int_{0}^{1} \Psi_{t x} \phi_{t}
$$

for some constant $\eta>0$.

Now, we handle the last term in the right-hand side of (2.15), using $(2.1)_{3}$ as follows:

$$
\begin{aligned}
\int_{0}^{1} \psi_{x t} \phi_{t}= & \frac{1}{\beta} \int_{0}^{1} \phi_{t}\left(\left(\kappa \theta_{x x t}+\delta \theta_{x x}-\rho_{3} \theta_{t t}\right)\right. \\
= & -\frac{\rho_{3}}{\beta} \int_{0}^{1} \phi_{t} \theta_{t t}-\frac{\delta}{\beta} \frac{\mathrm{d}}{\mathrm{d} t} \int_{0}^{1} \theta_{x} \phi_{x}+\frac{\delta}{\beta} \int_{0}^{1} \theta_{x t} \phi_{x} \\
& -\frac{\kappa}{\beta} \frac{\mathrm{d}}{\mathrm{d} t} \int_{0}^{1} \theta_{x t} \phi_{x}+\frac{\kappa}{\beta} \int_{0}^{1} \theta_{x t t} \phi_{x} .
\end{aligned}
$$

Multiplying by $\frac{\rho_{1} b}{K}-\rho_{2}$, we get

$$
\begin{aligned}
\left(\frac{\rho_{1} b}{K}-\rho_{2}\right) \int_{0}^{1} \psi_{x t} \phi_{t}= & -\frac{\mathrm{d}}{\mathrm{d} t}\left(\frac{\rho_{1} b}{K}-\rho_{2}\right) \int_{0}^{1}\left(\frac{\delta}{\beta} \theta_{x} \phi_{x}+\frac{\kappa}{\beta} \theta_{x t} \phi_{x}\right) \\
& +\left(\frac{\rho_{1} b}{K}-\rho_{2}\right)\left(\frac{\delta}{\beta} \int_{0}^{1} \theta_{x t} \phi_{x}+\frac{\kappa}{\beta} \int_{0}^{1} \theta_{x t t} \phi_{x}-\frac{\rho_{3}}{\beta} \int_{0}^{1} \phi_{t} \theta_{t t}\right) .
\end{aligned}
$$

Therefore, recalling Young' s inequality and (2.13), we get, $\forall \varepsilon_{3}>0$,

$$
\begin{aligned}
\left(\frac{\rho_{1} b}{K}-\rho_{2}\right) \int_{0}^{1} \psi_{x t} \phi_{t} \leq & -\frac{\mathrm{d}}{\mathrm{d} t}\left(\frac{\rho_{1} b}{K}-\rho_{2}\right) \int_{0}^{1}\left(\frac{\delta}{\beta} \theta_{x} \phi_{x}+\frac{\kappa}{\beta} \theta_{x t} \phi_{x}\right) \\
& +\varepsilon_{3} \int_{0}^{1}\left(\phi_{t}^{2}+\Psi_{x}^{2}+\left(\phi_{x}+\Psi\right)^{2}\right)+\frac{C\left(\frac{\rho_{1} b}{K}-\rho_{2}\right)^{2}}{\varepsilon_{3}} \int_{0}^{1}\left(\theta_{x t}^{2}+\theta_{x t t}^{2}\right) .
\end{aligned}
$$

where $C$ is a positive constant depending on $\delta, \beta, \kappa, \rho_{3}$ only. We then define

$$
L(t):=\mathcal{L}(t)+\left(\frac{\rho_{1} b}{K}-\rho_{2}\right) \int_{0}^{1}\left(\frac{\delta}{\beta} \theta_{x} \phi_{x}+\frac{\kappa}{\beta} \theta_{x t} \phi_{x}\right)
$$


to get, from (2.15) and (2.16),

$$
\begin{aligned}
L^{\prime}(t) \leq & -2 \eta E_{1}(t)+\varepsilon_{3} \int_{0}^{1}\left(\phi_{t}^{2}+\Psi_{x}^{2}+\left(\phi_{x}+\Psi\right)^{2}\right) \\
& -\left(N \kappa-\frac{C^{\prime}}{\varepsilon_{3}}\right) \int_{0}^{1} \theta_{x t t}^{2}-\left(N \kappa-\lambda-\frac{C^{\prime}}{\varepsilon_{3}}\right) \int_{0}^{1} \theta_{x t}^{2} .
\end{aligned}
$$

where $C^{\prime}=C\left(\frac{\rho_{1} b}{K}-\rho_{2}\right)^{2}$. Using (2.5), choosing $\varepsilon_{3}$ small enough and taking $N$ large enough so that $L$ is positive and $N \beta \kappa-\lambda-\frac{C^{\prime}}{\varepsilon_{3}}>0,(2.17)$ takes the form

$$
L^{\prime}(t) \leq-\eta E_{1}(t)
$$

Simple integration, recalling that $E_{1}$ is non-increasing, leads to

$$
t E_{1}(t) \leq \int_{0}^{t} E_{1}(s) \mathrm{d} s \leq \frac{1}{\eta}(L(0)-L(t)) \leq \frac{L(0)}{\eta}
$$

Consequently,

$$
E_{1}(t) \leq \frac{L(0) / \eta}{t} \leq \frac{k\left(E_{1}(0)+E_{2}(0)\right)}{t}, \quad \forall t>0 .
$$

This completes the proof.

Remark 2.9 Similar results can be established for boundary conditions of the form

$$
\phi(0, t)=\phi(1, t)=\Psi_{x}(0, t)=\Psi_{x}(1, t)=\theta_{x}(0, t)=\theta_{x}(1, t) .
$$

Acknowledgments This work is supported by KFUPM funded project \# SB100003.

Open Access This article is distributed under the terms of the Creative Commons Attribution License which permits any use, distribution, and reproduction in any medium, provided the original author(s) and the source are credited.

\section{References}

1. Ammar-Khodja, F.; Benabdallah, A.; Muñoz Rivera, J.E.; Racke, R.: Energy decay for Timoshenko systems of memory type. J. Differ. Equ. 194, 82-115 (2003)

2. Chandrasekharaiah, D.S.: Hyperbolic thermoelasticity: a review of recent literature. Appl. Mech. Rev. 51, 705-729 (1998)

3. Feng, D.X.; Shi, D.H.; Zhang, W.: Boundary feedback stabilization of Timoshenko beam with boundary dissipation. Sci. China Ser. A 41, 483-490 (1998)

4. Fernández Sare, H.D.; Muñnoz Rivera, J.E.: Stability of Timoshenko systems with past history. J. Math. Anal. Appl. 339, 482-502 (2008)

5. Green, A.E.; Naghdi, P.M.: A re-examination of the basic postulates of thermomechanics. Proc. R. Soc. Lond. A 432, 171-194 (1991)

6. Green, A.E.; Naghdi, P.M.: On undamped heat waves in an elastic solid. J. Thermal Stress. 15, 253-264 (1992)

7. Green, A.E.; Naghdi, P.M.: Thermoelasticity without energy dissipation. J. Elast. 31, 189-208 (1993)

8. Guesmia, A.; Messaoudi, S.A.: On the control of solutions of a viscoelastic equation. Appl. Math Comput. 206, 589-597 (2008)

9. Guesmia, A.; Messaoudi, S.A.: General energy decay estimates of Timoshenko systems with frictional versus viscoelastic damping. Math. Methods Appl. Sci. 32, 2102-2122 (2009)

10. Kim, J.U.; Renardy, Y.: Boundary control of the Timoshenko beam. SIAM J. Control Optim. 25, 1417-1429 (1987)

11. Messaoudi, S.A.; Mustafa, M.I.: On the internal and boundary stabilization of Timoshenko beams. Nonlinear Differ. Equ. Appl. 15, 655-671 (2008)

12. Messaoudi, S.A.; Mustafa, M.I.: On the stabilization of the Timoshenko system by a weak nonlinear dissipation. Math. Method Appl. Sci. 32, 454-469 (2009) 
13. Messaoudi, S.A.; Michael, P.; Said-Houari, B.: Nonlinear damped Timoshenko systems with second: global existence and exponential stability. Math. Method Appl. Sci. 32, 505-534 (2009)

14. Messaoudi, S.A.; Mustafa, M.I.: A stability result in a memory-type Timoshenko system. Dyn. Syst. Appl. 18, 457-468 (2009)

15. Messaoudi, S.A.; Said-Houari, B.: Energy decay in a Timoshenko-type system of thermoelasticity of type III. J. Math. Anal. Appl. 348, 298-307 (2008)

16. Messaoudi, S.A.; Said-Houari, B.: Uniform decay in a Timoshenko-type with past history. J. Math. Anal. Appl. 360, 459-475 (2009)

17. Muñoz Rivera, J.E.; Racke, R.: Mildly dissipative nonlinear Timoshenko systems—global existence and exponential stability. J. Math. Anal. Appl. 276, 248-276 (2002)

18. Muñoz Rivera, J.E.; Racke, R.: Global stability for damped Timoshenko systems. Discrete Contin. Dyn. Syst. 9, 1625-1639 (2003)

19. Muñoz Rivera, J.E.; Racke, R.: Timoshenko systems with indefinite damping. J. Math. Anal. Appl. 341, 1068-1083 (2008)

20. Mustafa, M.I.; Messaoudi, S.A.: General energy decay rates for a weakly damped Timoshenko system. Dyn. Control Syst. 16, 211-226 (2010)

21. Raposo, C.A.; Ferreira, J.; Santos, M.L.; Castro, N.N.O.: Exponential stability for the Timoshenko system with two weak dampings. Appl. Math. Lett. 18, 535-541 (2005)

22. Santos, M.L.: Decay rates for solutions of a Timoshenko system with a memory condition at the boundary. Abstr. Appl. Anal. 7, 531-546 (2002)

23. Soufyane, A.; Wehbe, A.: Uniform stabilization for the Timoshenko beam by a locally distributed damping. Electron. J. Differ. Equ. 29, 1-14 (2003)

24. Timoshenko, S.: On the correction for shear of the differential equation for transverse vibrations of prismaticbars. Philos. Mag. 41, 744-746 (1921) 